\title{
Rainbow of hope after the storm: Couples' experiences of pregnancy after stillbirth
}

\author{
Margaret M. Murphy
}

School of Nursing and Midwifery, UCC

\author{
"Hope" is the thing with feathers - \\ That perches in the soul - \\ And sings the tune without the words - \\ And never stops - at all - \\ EMILY DICKINSON
}

\section{Pregnancy loss: a common and an underestimated event that has far reaching consequences}

Despite phenomenal advances in maternity care over the past fifty years babies still die around the time of their birth and the causes may be varied. Pregnancy loss remains the most common complication of pregnancy today with one in five pregnancies ending in loss. These losses can occur at any stage from fertilisation through pregnancy to birth.

Stillbirth is when the baby, greater than 24 weeks gestation or weighing more than 500 grams, is born having never shown signs of life. Most of the estimated 2,000,000 stillbirths annually, happen in low and middle resource countries, and occur for a combination of reasons: lack of access to trained healthcare assistance around pregnancy and birth, poor nutrition, and a dearth of resources - to name but a few. Stillbirth remains an issue for high resource countries like Ireland also. In high resource countries, it is expected that one in two hundred pregnancies annually will end in stillbirth. Despite our best efforts these rates have remained static for the past ten years. On average there are three hundred and fifty stillbirths in Ireland every year. To put that into context that is a jumbo jet full of babies who never get to go home with their parents, who never get to fulfil their milestones, but these children are never forgotten about by their parents.

\section{The veil of silence that surrounds stillbirth and its effect on couples}

Loss and grief are universal issues, fundamental to the human experiences that affect us all at some point in our lives. Researchers have suggested that the loss of stillbirth is 
unique. The idea of death occurring before birth is difficult to grasp as it challenges our preconceptions of a safe world order. There are few shared memories or tangible proof of the baby's existence. The baby has not lived outside its mother's body and is to an extent 'unknown' by others. Therefore this loss is often unrecognised or underappreciated by a couple's social support network or wider society and has been referred to by Cacciatore in 2013 as 'silent grief'. This lack of recognition can have devastating health and social consequences for couples including depression, post-traumatic stress disorder, complicated grief, and relationship breakdown. But in the midst of despair there is hope too, a high percentage of couples will go onto have another pregnancy, often very soon after their initial loss. Many women are pregnant again within twelve to eighteen months after their stillbirth. Being pregnant again can prove challenging for the women and their partners. They are attempting to bond with their new baby; while actively grieving for the baby they have lost. Previous research has highlighted mental health issues in the pregnancy after loss such as increased levels of stress, anxiety, depression, post-traumatic stress disorder and even attachment difficulties with the baby born after loss. While it is recognised that stillbirth can be experienced by same sex couples and by single women the inclusion criteria for my study is heterosexual couples. It is these couples and their experiences of pregnancy after loss, that are the focus of my research.

\section{My research}

My midwifery background, supporting women and their partners pregnant again after pregnancy loss, led me to my research question. The midwifery profession is very much grounded in clinical practice and the experiences of working, in partnership, with women and their families. Following a thorough literature search I was aware that the research on this area to date had a very pathological or illness focus. Midwifery, conversely, has a much more salutogenic or health focus with its theoretical foundations in the social model of pregnancy and birth. Salutogenesis was first described by the medical sociologist Aaron Antonvosky in 1979 and comes from the Latin salus (health) and the Greek genesis (origin). It is a theory that focuses upon factors that influence health and wellbeing rather than illness. Therefore I wished to apply a more unique and health focused approach to my research. I questioned if the act of getting pregnant again after loss was in itself a hopeful one. Therefore my research question became, 'what is it like for couples to experience pregnancy again after the experience of stillbirth?' To answer this question I have conducted extensive interviews with women who are pregnant after stillbirth and their partners. 


\section{Understanding couples' experiences of pregnancy after stillbirth}

My research is unique as it is the first to examine the dynamics of couples as a unit or dyad in the face of pregnancy after loss in addition to its salutogenic perspective. Currently I am engaged in data analysis. Initial reading of the data has revealed the following: couples expressing the importance of having the challenges they face recognised, the importance of the provision of appropriate support and open communication, and that their deceased baby is honoured and acknowledged. Some tentative themes are emerging. Couples have reported wishing to honour the baby they have lost and the importance of protecting the time they see as 'theirs' (the deceased infants) before they can contemplate planning another pregnancy. The study offers new insights on how couples support and help one another through pregnancy after loss, in particular the unique role of fathers. Hope also appears to be present but without in depth analysis it is too early to speculate the role that it plays.

\section{Potential impact}

The potential impact of my research nationally and internationally is in its innovative focus on couples and on health rather than illness within this population. The practical application of my research will be in the development of concrete, supportive intervention protocols for women pregnant again after loss and their partners. These will help address many of the issues highlighted previously in the research such as stress, anxiety, difficulty in bonding and relationship issues.

"As long as we are being remembered, we remain alive" Carlos Ruiz Zafón

Margaret M. Murphy is a College Lecturer and a final year Doctor of Nursing student under the supervision of Dr Patricia Leahy-Warren, Senior Lecturer and Professor Eileen Savage, Head of School and Chair in Nursing, School of Nursing and Midwifery, Brookfield Health Sciences Complex, University College Cork, Ireland.

A special word of thanks to the couples who shared their stories so honestly and openly. 